\title{
FTRS: A Mechanism for Reducing Flow Table Entries in Software Defined Networks
}

\author{
Bing Leng ${ }^{\mathrm{a}, \mathrm{b}, *}$, Liusheng Huang ${ }^{\mathrm{a}, \mathrm{b}}$, Chunming $\mathrm{Qiao}^{\mathrm{c}}$, Hongli Xu $\mathrm{Xu}^{\mathrm{a}, \mathrm{b}}$, Xinglong \\ Wang $^{\mathrm{a}, \mathrm{b}}$ \\ a96 Jinzhai Road, School of CS \& Tech., USTC, Hefei, 230027, China \\ ${ }^{b} 188$ Renai Road, Suzhou Institute for Advanced Study, USTC, Suzhou, 215123, China \\ ${ }^{c}$ State University of New York, Buffalo NY, 14260-2000, USA
}

\begin{abstract}
A flow table in an OpenFlow based switch is used to not only operate the switch, but also carry out the control function of the Software Defined Network (SDN) controller. However, as a core participant of network management, its limited storage has been a significant roadblock for the industrialization of SDN. It is a significant problem to reduce the number of flow entries needed in the almost full-filled flow tables, and at the same time, to retain the original QoS as much as possible - or known as the "Flow Table Congestion Problem" (FTCP). We propose a mechanism called "Flow Table Reduction Scheme" (FTRS) to efficiently solve FTCP and evaluate the performance of FTRS both via simulation and experiment. The results show that FTRS is able to reduce the number of flow entries by $98 \%$ at most of the size of flow table with no influence on network's normal functions.
\end{abstract}

Keywords: Software Defined Network, flow table, reduction, QoS.

\section{INTRODUCTION}

In traditional networks, a switch is not only responsible for basic packet switching/forwarding, but also makes decisions such as route selection, etc.

\footnotetext{
* Corresponding author

Email address: lengb@mail.ustc.edu.cn (Bing Leng)

The original vision of this paper [1] is shown in IEEE/ACM International Conference on Communications (ICC) 2015.
}

Preprint submitted to Journal of ${ }^{A} T_{E} X$ Templates

March 9, 2017

(C) 2017. This manuscript version is made available under the Elsevier user license http://www.elsevier.com/open-access/userlicense/1.0/ 


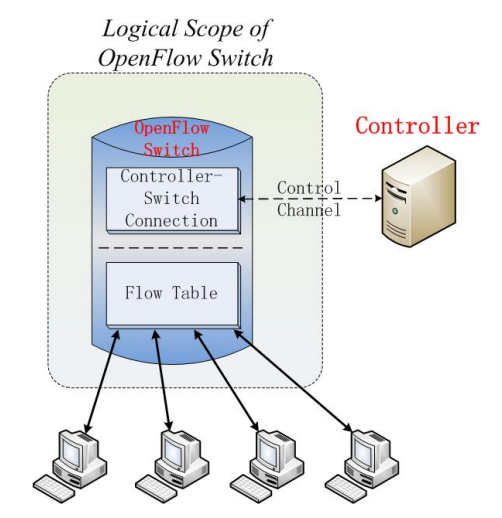

Figure 1: A Logical Scope of An OpenFlow Switch

Though convenient, such a mode of operation is considered not flexible or fast 5 enough to meet the increasing demand on the network management by many real-time and high-speed applications. This stimulates the recent development of software defined networks (SDN)[2]. SDN is designed to separate the control function from the packet transmissions, which reduces the complexity of the switches and increases the control flexibility of the network.

There are two logical elements in a SDN - a SDN network controller and a forwarding unit, the latter often referred to as an Openflow switch. The $S D N$ controller is responsible for designing the rules of the network, and the OpenFlow switches forward the packets according to these rules. In particular, the OpenFlow switches report flow traffic information they detected to the controller, where the response rules are determined and translated into distributed flow management entries. These entries are then transfered to the corresponding switches and stored in their flow tables. Each OpenFlow switch will then forward packets according to the entries stored in its flow table and also report to the SDN controller the statistical information about each flow. In this way, 20 the controller possesses the full control of the network management and the 
switches could achieve fast forwarding as well. A logical view of an OpenFlow switch is shown in Fig 1.

According to the design of SDN, it is obvious that the flow table plays an important role in SDN's functionality and QoS guarantee. Basically, SDN provides three basic functions, namely the routing management, manual control of network and ability to carry other applications. However, in practice, the flow table suffers from the limited storage due to the use of TCAM [3] — on which the flow table is realized in the majority of existing equipments in industry to achieve fast forwarding - and do harm to all these three QoS requirements.

so For example, the commercial OpenFlow switches used in our experiment possess only 3000 flow entries in the flow table, and are not enough to support a LAN with 30 terminals for 3 hours [1]. As a result, after the flow table is full filled, the basic routing, dynamic management and service providing for other applications may all be failed due to the fact that the flow table is the low35 est level in SDN functionality. The reason of the fast flow entry occupation is due to the fine-grained flow entry storage of SDN. The fine-grained flow entry storage indicates that a flow is recognized with the intersection of the most confirmed attributes that can be recognized by a flow entry. On one extreme, two adjacent packets with everything the same except for the port number will be recognized belonging to two different flow entries. In order to solve this problem, it is intuitive to make the combination to compress the fine-grained entries into fewer coarse-grained ones. Namely, we can use mask code technology to combine the original diverse fine-grained flow entries into a new coarse-grained one with a slightly larger matching range to reduce the number of entries at the cost of losing some precise matching information. The format of standard SDN flow entry permits such action, which we will introduce later. But on the other hand, if a flow is routed by combined entries all the way along its route path, the SDN controller is unable to obtain its statistical data, which is important and necessary to the SDN functionality. As a result, we have to make a careful decision on whether/how to combine these multiple flow entries with significant compression performance and few original flow entries lost. Moreover, since the 
entry compression is based on the combination, it is possible that an originally unfamiliar flow to the switch may be matched and routed by the newly generated coarse-grained entries. This should also be forbidden in order to avoid potential routing mistakes.

In this paper, we address the above dilemma between the need to reduce the number of flow entries but at the same time, the need to preserve the QoS of SDN functionality, which is also referred as "Flow Table Congestion Problem" (FTCP). Previous works fail in solving it due to either high reduction performance $[4,5,6,7]$ or the QoS violation $[8,9,10]$. For example, the work in [4] and [5] achieved the reduction in the number of rules generated at the controller by giving up a part of the controller's functions, which results in the reduction in the number of flow entries. The work in [6] and [11] focused on rules deployment and they contributed little to the reduction in the number of flow entries. There were several studies on the firewall rule reduction problem $[9,10]$, but their solutions are not suitable for flow entry reduction in SDN for several reasons to be elaborated in Section II. Since FTCP is a significant roadblock of practical SDN application, it is both important and timely to search for effective solutions.

The requirements of solving FTCP can be summarized as follows:

1 Consistency: All the flows should be routed the same way before and after the reduction.

2 Absoluteness: All manually added rules must be matched and executed as the first priority.

3 QoS: The corresponding entry of each flow should be remained at least in one switch after the reduction.

4 Extended Routing Avoidance: The originally new flow should not be matched by the compressed entry after reduction.

With these requirements, we design an efficient scheme called Flow Table

Reduction Scheme (FTRS for short) to solve FTCP. FTRS reserves the existence of each flow entry at the edge of the flow path and compresses it with 
other similar entries in the middle. The rationality and reasons of doing so are also discussed during the introduction of FTRS. The combination of the flow entries is achieved on the base of its structure. This is determined by the OpenFlow Protocol [12] and we manage to achieve a high compression percentage by carefully selecting the reduction attributes. We test FTRS using both simulation and a small-scaled experiment. The results show perfect reduction performance of FTRS. The major contributions of work are listed below:

- To the best of our knowledge, this is the first time that the Flow Table Congestion Problem is addressed with the above four requirements involved.

- The average number of flow entries in the flow table of core switch drops by more than $95 \%$ generally in LAN environment according to our simulation and experiment

95

- The functions of SDN such as routing management and statistics data collection are unaffected (or even improved)

- Manual operations on SDN are allowed in FTRS with no influence on network functions

The rest of this paper is organized as follows. Section II reviews the flow 100 table and related work. The problem to be addressed, FTCP, and solution requirements are discussed in Section III and Section IV presents the proposed FTRS algorithms and architecture. The results from the simulation and experiment are discussed in Section V. In Section VI we draw some conclusions.

\section{BACKGROUND}

In this section, a brief introduction of flow tables in SDN using OpenFlow is given first, following by a review of related work including rule reduction in firewalls. 


\begin{tabular}{|l|l|l|l|l|l|}
\hline Match Fields & Priority & Counters & Instructions & Timeouts & Cookies \\
\hline
\end{tabular}

\section{(a) Storage of An Flow Entry}

\begin{tabular}{|c|c|c|c|c|c|c|c|c|c|c|c|}
\hline $\begin{array}{c}\text { Ingress } \\
\text { Port }\end{array}$ & Ether & Ether & Ether & Vlan & Vlan & IP & IP & IP & IP ToS & TCP/UDP & TCP/UDP \\
Dst & Type & id & Priority & src & dst & proto & bits & Src Port & Dst Port \\
\hline
\end{tabular}

\section{(b) Detailed Storage of Match Fields}

Figure 2: Format of the Flow Entry Defined in OpenFlow[12]

\subsection{Flow \& Flow Table}

The most typical characteristic of SDN is its innovation on separating the control plane away from the data plane in switches - they are only responsible for local high speed data transmission without making any decision. The flow table is the managing medium between the controller and the switch to accomplish rule expression, routing management and fast forwarding.

Instead of packet level, OpenFlow switches recognize and forward the data at flow level. A flow is a set of packets that possess some properties in common. For example, packets are thought to be components of a certain flow if they are sending sequentially and have the same source and destination addresses. The definition of the flow is flexible, which gives flow management endless possibilities.

According to the design of the most widely used SDN protocol OpenFlow[12], the flows are recognized and routed by the flow entry stored at the OpenFlow switch. The standard format of the flow entry is shown in Fig. 2. The Match Field is used to recognize and identify the flows, according to their attributes such as ingress port, IP source and destination addresses, Internet protocol, vLAN, etc. These attributes could be null, and the interaction of the non-null ones determines which flow should be routed by the entry. In this case, they should not be all null. Since there are plenty of flows going through the switch 
all the time, there are numbers of entries stored at the switch at a time, and their combination is a structure called flow table, in which the Priority item of the entries determine their matching sequence. When a flow is recognized by an entry, it should take actions as the Instructions item indicates, and if an entry is not matched for a longer time than its Timeouts value, it is deleted automatically.

Then a flow in SDN could be defined as a sequence of continuous packets that are recognized by the same flow entry described as Fig. 2. Instead of packets, SDN equipments manage network transmissions of flows.

It is a core promise of SDN that to separate the control functions away from the routing functions at the switch. As a result, the flow table at the switch is managed and maintained by the SDN controller instead of itself. The switch is mainly responsible for recognizing the flows in its flow table and make quick switching/routing. When a flow is not matched by any entry in the flow table, it is recognized as a new flow and reported to the SDN controller, at where its attributes and routing path are calculated. Then, the SDN controller updates all the flow tables at the switches along its routing path to realize the transmission. The flow table is not only the foundation of switch routing, but also the functioning medium between the OpenFlow switch and the SDN controller, indicating it's the most important component in SDN management and applications.

\subsection{Boundary and Core Switches}

In a representative topology shown as Fig. 3, there are switches linked directly with terminals and ones routing the flows among the other switches. This is especially common in nowadays LAN deployment and data center networks. Generally speaking, there won't be too many terminals linked with one switch due to the limited number of physical port. As a result, this kind of switch won't be bothered by too many flows since it is only responsible for servicing the terminals linked with it. We call this kind of switch the Boundary Switch. On the other hand, a switch may encounter much more flows when it serves 


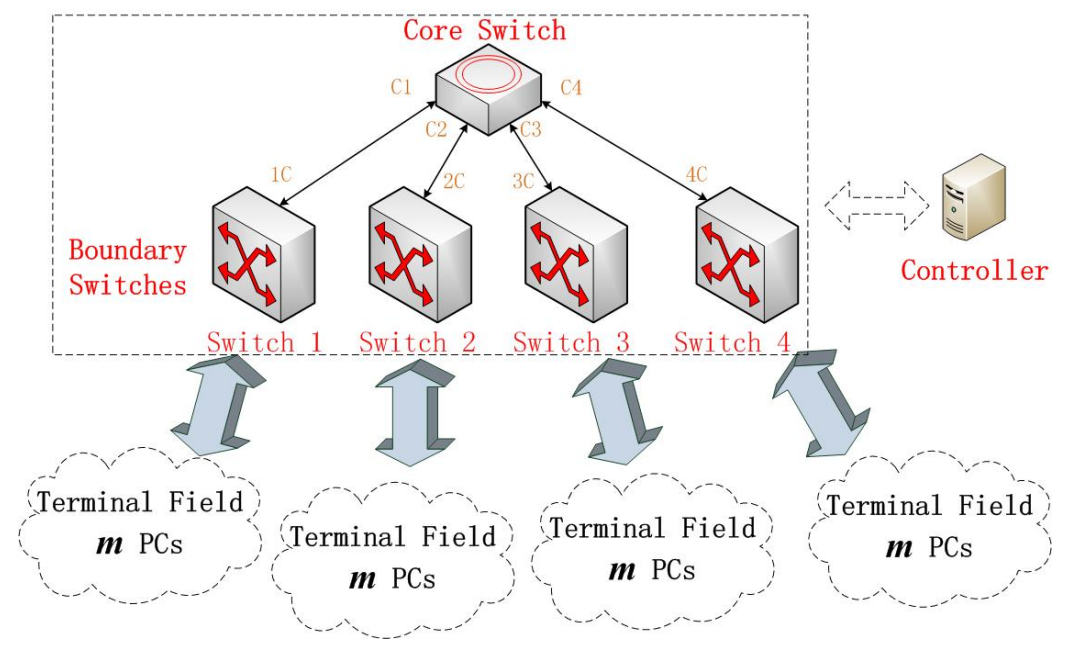

Figure 3: An Example of SDN Topology

other switches, and obviously, its flow table is much more likely to be full-filled. We call this kind of switch the Core Switch. Since there is fundamental difference between the occurrence probability of FTCP at the core and boundary switches, we focus on compressing the flow entries at the core switch, and meanwhile, maintaining the ones at the boundary switch. Consequently, we are able to solve FTCP with the QoS of network guaranteed.

\subsection{Proposed Network Architectures}

The limited storage of flow table is a significant bottleneck of SDN, and several significant works $[4,5,6,11]$ mentioned the Flow Table Congestion Problem and come up with their unique solutions.

Devoflow [4] proposes a method for high performance in SDN. Its primary attention is paid on the limitation brought by the controller's poor performance. By separating the management of small flows from the giant flow, Devoflow 
reduces the probability of communication between controller and switch, and the number of flow entries is reduced as a coproduct. However, the properties of some flows that should be recorded are unavailable to the users due to the lack of small flow information in controller.

[5] contributes little in global flow table reduction. In [5] one authority switch is responsible for connecting with the controller and splitting rules made by the controller. The authority switch stores all the forwarding rules to reduce the number of rules stored in other switches, which leads to redundancy flow rules storage on the contrary. Meanwhile, the limited number of core switches is the premise of table splitting. Since core switches are geographically close to each other, their flow tables will have much in common, which is the key point of table splitting. If there are few core switches or the core switches are far away from each other, table splitting may suffer bad performance.

The rule placement problem is presented by Palette [6] and One Big Switch [11]. They try to minimize the number of rules that will be deployed in switches when the controller is calculating a path for a new flow. They focus on reducing the number of flow entries when the flow is set up instead of looking for the reduction opportunities in the current flow table, which means their achievement is the best supplement to our scheme.

\subsection{Flow Table Aggregation}

Recently, several researches have been proposed on the topic of "Flow Table Aggregation (or Aggregation)" [13, 14], which asks the controller to do the decision as long as it receives a new OF rule - whether to aggregate it with existing ones or not. [13] proposes the concept of Aggregation, but ignores all the QoS requirements. [14] requires the rules to be aggregated should share as long routing path as possible, and the controller should calculate the QoS requirements first. As a result, its aggregation efficiency is barely satisfactory [15]. The Aggregation calculation is taken place in a rule level, which indicates these above proposes are upper-layer solutions. Their operation cost is high because they are on-demand approaches. Each new rule should be calculated 
with all the existing ones and the aggregation process may cause routing interruption. Our approach - on the other hand - is the under-layer solution, and achieves QoS requirements by maintaining the Terminal Entries (which will be introduced later). The reduction is processed on each Core Switch and hence almost achieves much better efficiency. Meanwhile, we manage to maintain the consistency of the flows by carefully designing the flow table update method.

\subsection{TCAM Optimization}

The main reason of limited flow table size is the relatively small TCAM storage, hence there are also some researches focusing on optimizing the TCAM efficiency. [16] proposes a solution based on combining the SRAM memory, which is significantly cheaper, with the TCAM. More specifically, they argue that the simple packet processing such as MAC and VLAN based forwarding, can be done in SRAM while more complex processing involving wildcarding can be done using TCAM-based flow tables. In this way, the need for having a large TCAM is reduced. Another work suggesting a mixture of SRAM and TCAM is [17]. An algorithm that combines several finer grained wildcards into fewer coarser grained wildcards is proposed in order to reduce the number of OF

entries. This solution is applied in the context of server load balancing hence the paper does not provide tests and results to assess the impact on the degree of reduction in the flow table space.

\subsection{Rules Reduction in Firewalls}

The study issue in firewall rules reduction has some similarities with our study, but several reasons prevent them from solving the problem.

[9] depicts the detailed formulation of the rules reduction in firewalls, and [10] presents several solutions with different concentrations. But none of the models or the solutions is suitable for reducing the flow tables in SDN for the following four reasons:

1. The rules in firewall need to be Complete [9] which is performanceinfluenced and not practical in OpenFlow switches. 
2. The objective of rules reduction in firewalls makes the solutions unable to collect information of all the flows respectively.

3. Firewalls are not adjacent according to the formulation (even then they are considered as one logical firewall), but the main focus of this paper is the core switches who are linked with other switches.

4. Rules conflict is forbidden in firewalls but is allowed and a common technique for reducing the flow entries in our mechanism.

It is obvious that our problem could not be solved with rules reduction methods in the researches of firewalls.

\section{PROBLEM STATEMENT}

In this section, we present a significant problem called FTCP which is revealed during the usage of SDN in industry, explaining its causes, importance and urgency of being solved.

In order to prove how the size of flow table influences the switch performance, we use H3C S5820V2 OpenFlow switch [18] with an Opendaylight [19] as a controller to test the average response time of matching/adding/deleting/modifying entries. Each operation is tested with randomly selected entries among increasing flow table size for 100 times, and the average time cost is shown in the Table $? ?$.

Table 1: Time Cost vs. Flow Table Size

\begin{tabular}{|c|c|c|c|c|}
\hline FT Size & Match & Add & Delete & Modify \\
\hline 100 & $1.71 \mathrm{~ms}$ & $11 \mathrm{~ms}$ & $10 \mathrm{~ms}$ & $6 \mathrm{~ms}$ \\
\hline 500 & $5.2 \mathrm{~ms}$ & $171 \mathrm{~ms}$ & $87 \mathrm{~ms}$ & $33 \mathrm{~ms}$ \\
\hline 1000 & $15.7 \mathrm{~ms}$ & $290 \mathrm{~ms}$ & $112 \mathrm{~ms}$ & $51 \mathrm{~ms}$ \\
\hline 2000 & $27.3 \mathrm{~ms}$ & $454 \mathrm{~ms}$ & $207 \mathrm{~ms}$ & $107 \mathrm{~ms}$ \\
\hline 3000 & $41.3 \mathrm{~ms}$ & $15589 \mathrm{~ms}$ & $573 \mathrm{~ms}$ & $133 \mathrm{~ms}$ \\
\hline
\end{tabular}

As shown in the table, the average time cost increases along with the flow table size. Since the flow table of H3C S5820V2 OpenFlow switch only possesses 
3000 entries, it costs much more time to add a new entry when the flow table is full. Also, the adding operation fails occasionally, which is not revealed in the table. Although the time cost may be acceptable according to the table, the fact that the flow table is easily to be fully filled in a short time is the more dominating reason for us to do the reduction.

As we discussed in Fig. 2, the flow is recognized by the intersection of the attributes stored in the Match Field of the flow entry. The format of the flow entry leads to a representative character of SDN — the fine-grained management and storage. Particularly, even flows with the same source and destination addresses but different ports are treated as different flows in SDN. This mechanism contributes to achieving perfect network managing performance and serving applications, but with no doubt burdens a lot to the flow entry storage at the same time.

In order to give a clear description of FTCP, a small scale network topology shown as Fig 3 is used as an example in the following. There are 1 SDN controller, several OpenFLow switches and plenty of personal computers (terminals) in the network. In order to achieve a vivid understanding of the problem with universality, we formulate the example in Fig 3 as follows:

1 There are $k$ switches connected to the central Core Switch.

2 Without loss of generality, the Core Switch is directly linked with $n$ terminals (usually none), while the other adjacent switches are responsible for routing messages for $m$ terminals linked directly to them. Due to the location of these switches in the network, they are so called the Boundary Switches.

3 The data pipelines between the switches conform the law that the port ij of switch $i$ connects to the port $j i$ of switch $j$ (e.g. port $1 C, C 1$ in the figure). All the OpenFlow switches are managed by the SDN controller.

If we want to reduce a set of entries, we could select ones that possess the same Instructions and calculate the union of their Match Field. In this case, the normal data transmission is not influenced. However, since the SDN controller 
is also responsible for data collection, the independent transmission data of these entries are not available because they are collected together by the new combined entry.

As we mentioned earlier, the flow entry is installed in the flow tables of all the switches along the flow path. As a result, the entries could be divided into two parts according to the location of the switches. Generally, the switches at the both edge of the flow path are more important since their collected data could be used to calculate the quality of service. On the other hand, the entries in the switches in the middle of the flow path are less important and few applications care them. In this case, we classify the flow entries on the location of the switches along the flow path and name them the Terminal Entries and Medium Entries respectively.

Considering Switch 1 in Fig. 3, there are $m$ terminals linked directly to it. We call this switch a Boundary one because it locates at the boundary of the network and mainly services its own terminals. The flow entries in the flow table of the Boundary switches are mostly ones related to the terminals linked directly to the switch. Although stored at fine-grained level, the number of such entries are limited by the number of physical ports on the switch and in turn not too many. On the other hand, the Core Switch shown in the figure is responsible for retransmitting the flows passing through it and as a result, it is relevant with all the terminals in the network. The number of flow entries in its flow table will explode on the base of the fine-grained storage and the network size. This indicates the Core Switch should be more vulnerable than the Boundary ones on the storage crisis of flow entry and we are going to prove it in the following.

Firstly, we simplify the problem by ignoring the fine-grained storage of the flow entry. We assume that all the terminals in Fig. 3 generate one flow for each of the rest and the total number of switches is $k+1-k$ Boundary ones and 1 Core Switch. Without loss of generality, we also arrange $n$ terminals connected directly to the Core Switch. Considering a Boundary Switch as an example, 310 it possesses $m(m-1)$ entries for servicing the data transmission within its $m$ terminals and $2((k-1) m+n) m$ entries for the communication between its $m$ 
terminals and the rest $(k-1) m+n$ ones. On the other hand, for the Core Switch, it should also possess $n(n-1)$ entries for the communication within its $n$ terminals and $2 \mathrm{kmn}$ ones for the communication between its $n$ terminals and the rest $k m$ ones. Meanwhile, there should be $k(k-1) m^{2}$ entries for realizing the data retransmission on it. We take 5 sets of values of $(k, m, n)$ that are mostly common in practical LAN and calculate the number of flow entries in the Core and Boundary switches respectively.

Assuming one terminal (e.g. computer $A$ ) connected to Switch 1 needs to send a flow to another terminal (e.g. computer $B$ ) connected with Switch 2 , it is easy to calculate the flow path as $A \rightarrow$ Switch $1 \rightarrow$ Core Switch $\rightarrow$ Switch $2 \rightarrow$ $B$. There are three flow entries for this flow to be installed into the flow tables of Switch 1, Switch 2 and Core Switch separately. Typically, Switch 1 and 2 are at the edge of the flow path, while the Core Switch is in the middle. In this case, the corresponding two flow entries in Switch 1 and 2 are Terminal ones, and one in the Core Switch is a Medium one. It is the same when the transmission is between two terminals connected to the same switch, and since the switch is both the start and end edge of the flow path, the corresponding flow entry is undoubtedly a Terminal one. According to our previous discussion, if an entry is a Terminal one, it should be remained after the reduction. If an entry is a Medium one, it could be used as a resource for combination. The contradiction of Terminal and Medium entries is exhibited in Table 2.

Table 2: Terminal \& Medium Entry

\begin{tabular}{|c|c|c|}
\hline Property & Terminal Entry & $\begin{array}{c}\text { Medium } \\
\text { Entry }\end{array}$ \\
\hline Joinable & Negative & Positive \\
\hline Mostly Appearance & Boundary Switch & Core Switch \\
\hline Origin & Controller \& Manual Operation & Controller \\
\hline Next Hop & Terminal \& Switch & Switch \\
\hline Amount in Core Switch & $n(n-1)+2 k m n$ & $k(k-1) m^{2}$ \\
\hline
\end{tabular}


We also calculate the number of both types of the entries in the previous example, which is shown in Table 3 as the entries classification in the Core Switch. Notably, the entries in the Boundary Switches are all Terminal ones, while ones in the Core Switch are mostly Medium ones. This indicates the reduction is going to be happened in the Core Switch, which exactly possesses much more less important entries. In other words, the classification is suitable for entry reduction and solving FTCP.

Since the network condition may vary due to huge amount of reasons (such as load balancing policy, routing selection algorithms, recovery from unexpected emergency, manual management, etc.), we only take the most simple model in our analysis - namely there is only one link between each pair of terminals. However, the number will be much larger and the situation will be much more complicated in practical, which means our approach may achieve better performance than our simulation and test-bed experiment due to the underestimation of flow table size. All the formulas shown in Table 2 and 3 are calculated under this basic situation, and our main purpose is to show that even in the simple situation the flow table also faces severe congestion problem.

Notably, It is a promise of SDN to give the network administrator the ability to manage the routing rules/entries. This can be achieved manually as we mentioned in the Introdution Section. In each controller, APIs for manually modifying rules are provided. The network administrator can easily add/delete/modify any entry in the flow table of any switch as long as he wants to by calling these interfaces. For the manual rules - especially the manually added ones - our approach is also able to reduce them without harm to the administrator's purpose. This is because these manual rules are modified by the provided APIs of the controller - namely as to the controller, they are only the same as the other automatically added rules except for their high priority. Our approach leaves the terminal entries for the managing purpose and reduces the medium ones for better performance - no matter it is an automatically added rule or a manually added one.

Notably, in the table the number of entries easily exceeds several thousand 
Table 3: Number of Flow Entries with Different Parameters

\begin{tabular}{|c|c|c|c|c|c|}
\hline \multicolumn{3}{|c|}{ Parameters } & \multicolumn{3}{|c|}{ Flow Entries } \\
\hline \multirow{2}{*}{$k$} & \multirow{2}{*}{$n$} & \multirow{2}{*}{$m$} & \multicolumn{2}{|c|}{ Core Switch } & \multirow{2}{*}{ Boundary Switch } \\
\hline & & & Terminal & Medium & \\
\hline 4 & 0 & 10 & 0 & 1200 & 690 \\
\hline 4 & 0 & 15 & 0 & 2700 & 1560 \\
\hline 4 & 5 & 15 & 620 & 2700 & 1710 \\
\hline 6 & 0 & 15 & 0 & 6750 & 2460 \\
\hline 6 & 0 & 20 & 0 & 12000 & 4380 \\
\hline
\end{tabular}

and there are more entries in the Core Switch than the Boundary one.

Moreover, all the number calculated above are on the base of IP-level routing. Actually, SDN is functioning in a more fine-grained way. If we take the other storage into account (such as port, protocol, vLAN, etc. as shown in Fig. 2 ), the number of entries will be multiplied. Typically, some applications use plenty of ports for data transmission, such as QQ, which is the most popular chat application in China. The packets it generated are always with different ports for safety concern and are treated as component of different flows, which in turn aggravate the storage crisis.

The contradiction between the limited flow entry storage and the large amount of required ones is named as the "Flow Table Congestion Problem" (FTCP) in this paper. FTCP is a significant problem since the SDN functions are mostly realized on the base of the flow entries, and if there are not enough entries, the efficient management is impossible and even leads to a network collapse. Meanwhile, FTCP is hardly to be solved at the hardware level in short period because the flow table is realized by TCAM[3], which is famous for its fast processing, limited storage and high price.

FTCP has been sharply presented in industry. For example, the commercial OpenFlow switches we used possess only 3000 entries in its flow table[20]. It is a representative value among all the common commercial OpenFlow switches 
except for OVS[21] — a virtual switch simulation software. The switch with the

least flow entries is NEC PF5820, which only possesses 750 entries in its flow table and there is not any hardware SDN equipment that possesses more than ten thousand entries according to our knowledge. Although OVS avoids FTCP for its unlimited flow table size in theory, it is mostly used in Data Center since it lacks the ability to deal with routing and processing, which couldn't meet the promise of SDN in most cases.

To conclude, FTCP impacts the network performance of SDN badly, especially the Core Switches of the network, and without solving it, the popularization of SDN is unavailable.

\section{Flow Table Reduction Scheme (FTRS)}

In order to reduce the number of existed flow entries, it is an intuitive thought to combine several entries into fewer new ones. However, most of the SDN functions are achieved on the base of flow entries, such as the transmission management, flow recognition, data collection, etc. As a result, every flow entry is also important to SDN management. On the contrary, if the flow table is full (namely it encounters FTCP), the switch is not able to deal with new flows. The consequence may as light as transmission failure, and as serious as network collapse. To sum up, we should make a trade-off between the complete SDN functionality and the robustness of the network service.

In this section, we present a scheme called Flow Table Reduction Scheme ${ }_{05}$ (FTRS) to reduce the number of exited flow entries by combining the entries that are less important into fewer new ones. The specification of FTRS is introduced in the following.

Since the Algorithms take too much space, they are listed in the Appendix at the end of the paper.

\subsection{Optimizing Switch Selection}

As we mentioned in Section 3, our approach will only help reduce the flow table of Core switches. Although they possess more entries and are much more 
vulnerable to congestion, the Boundary Switches may also possess terminal entries which are suitable for reduction. But in our approach we leave them alone, this is due to the following two reasons:

1 The Boundary Switches are less likely to encounter flow table congestion and in turn to blame for the network errors/failures. Hence we prefer to reserve their flow entries to maintain the fine-grained management.

2 With the purpose of leaving the terminal entries undisturbed, the reduction must be processed only among medium entries. This may lead to unpredictable conflicts between reduced entries and the remained terminal ones and may also result in extreme extention of entry matching.

\subsection{Attribute Selection}

Our focus is find a best way to compress fine-grained flow entries into coarsegrained ones without any impact on the normal functions of SDN to solve the Flow Table Congestion Problem. Namely, the reduction percentage of the flow entries is the main attribute to evaluate the performance of FTRS, but on the other hand, the functions of the controller should be remained, which is revealed as the terminal entries should be available to the controller. In this case, our main idea is to compress the flow table of the Core Switch. Because the Core Switch suffers from FTCP the most and its flow entries are also stored in the flow table of at least one Boundary Switch.

Within the Match Field of the flow entry, each combination of attributes is able to be used as criterion to reduce the number of flow entries in FTRS.

${ }_{435}$ Since we classify the flow entries into two types, each individual flow entry is existed at least once in all the switches. In this case, the Medium entries are all free to be reduced. In order to achieve the best reduction performance, it is intuitive to select individual attribute for reduction. The selection depends on the characteristic of the network, the purpose of SDN management, etc. In most cases, the IP address involved attributes are the most suitable according to the network functioning characteristic. Different IP addresses indicate different network terminals and in turn different positions in the network topology. In 
this case, we select the IP address involved attributes as the default criterion in FTRS.

Except for IP address involved attributes, the rest ones are also useful in some specific situations. For instance, in a vLAN[22] environment, the IP addresses of terminals are mostly randomly arranged and share little in common. In this case, to select IP addresses involved attributes may experience poor performance. How to select criterion under such situation is also an important issue and should be based on the local characteristic of the network and application. In this paper, we take the Ingress Port as a representation to present the performance of FTRS in vLAN. This is because according to our vLAN experiment, the port attribute has the best distribution among all the generated flow entries and achieves the best performance in FTRS.

In this paper, we test the performance of FTRS on various attributes to select the best one for reduction. Generally, it is unnecessary to use FTRS on different attributes for several times because with enough empty storage available, it is encouraged to store fine-grained entries again. But in this paper, we always select one with the best performance as the criterion to illustrate the efficiency of FTRS.

\subsection{The Binary Trie}

As we mentioned above, our combination method is to combine the Match Field of the flow entries that possess the same Instructions. Then, the key question of our combination is how to seek the similarity among the Match Field of the entries for combination. In order to achieve the best reduction performance, we take the advantage of the inner characteristic of information storage - the binary system. Then, the similarity among the Match Field of the entries could be discovered as the matching of character string.

In FTRS, we use the binary trie[23] to seek for the attributes similarities. Within the binary trie, each node possesses three terms: the first one for the selected criterion, the second for the priority and the third for the Instructions value (indicating the actions after matching) of the flow entry. The location of 
the node represents the corresponding attribute string of selected criterion, and we could make reduction then if we could replace a sub-tree in the binary trie with a coarse-grained node.

In this case, each flow entry can be represented by a node in the binary trie. Moreover, it is obvious that a node possesses preciser matching conditions than its parent. As a result, the matching conditions of corresponding flow entry is a subset of that of its parent, the priority should be involved to determine whether a flow entry can be matched. Since the flow entries are examined sequentially, we also assign the priority value to the nodes in the binary trie. In this case, as long as a node is discovered to have a more prior ancestor (not restricted to parent), its corresponding flow entry will never be matched since the flows are matched by a prior entry (its ancestor). With the purpose of simplifying calculation, we can delete the node in the trie.

\subsection{FTRS on IP address}

FTRS sets up a binary trie root at first. Then, it traverses the flow table in a linear way, and generates corresponding leaves and their paths to the root in the trie according to the IP address (or the other attributes such as source port, destination port and priority in the Match Field of the Medium entry). There is a one-to-one correspondence between a non-empty node and a flow entry. After the traverse, we could get a binary trie $T_{I B}$ with the height of 32 - which represents the length of IPv4 address. The initialization of $T_{I B}$ is shown in Algorithm 1. Within $T_{I B}$, the positions of the nodes in the trie represent the values of their corresponding bit in the IP address and all the possible addresses are able to be expressed in a complete binary trie. A non-empty node may not be a leaf if the corresponding entry is a coarse-grained one. Then, if a node is discovered within the sub-tree rooted by another non-empty one, we should compare the priority of both nodes in advance. It should be guaranteed that a non-empty node possesses the highest priority within the sub-tree rooted by it, otherwise its children with lower priority will not be matched and should be deleted. Finally we will modify the binary trie to remove all the empty 


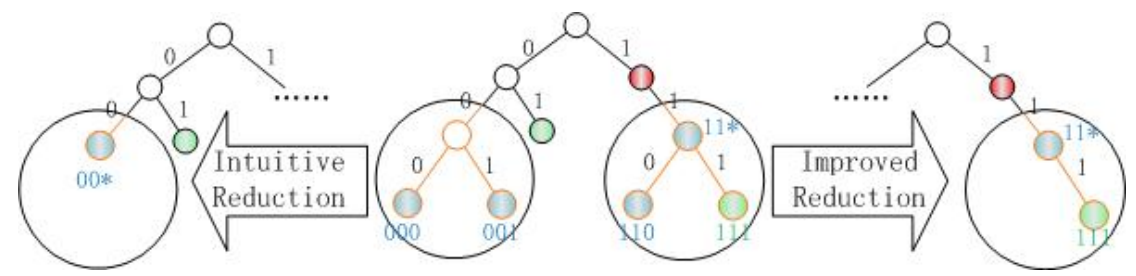

(a) Basic Idea of Intuitive \& Improved Reduction

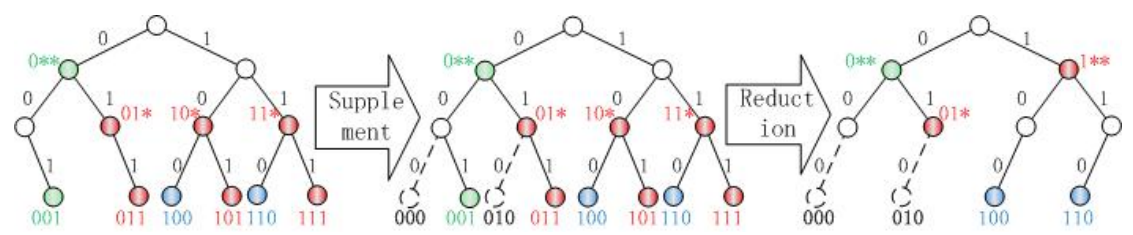

(b) Process of Improved Reduction Algorithm

Figure 4: Detailed Process of Reduction Algorithms with an Example of 3-digit

Addresses

nodes. This prune process is achieved by calling Algorithm 2 "Binary-TrieFixer $\left(\operatorname{root}\left[T_{I B}\right], \infty\right)$ ".

In order to provide a vivid presentation of FTRS, we use colors to generate the various kinds of Instructions of the Medium entries. Assuming part of the Medium entries in the flow table of the Core Switch shown in Fig 3 are listed as Table 4, they share the same prefix without loss of generality. The flow entries are matched on IP dst attribute. The priority indicates their matching sequence in the flow table and the entries share one color if their Instructions are the same. Now we get a Binary Trie shown as the middle graph in Fig 4(a).

Our focus is to replace the non-empty sub-trees with coarse-grained nodes. It is easy to come up with an intuitive thought for solving the problem, which is widely used on the route matching problem in the industry and exhibited as the Algorithm 3.

Considering the third and fourth flow entries in the Table 4, they share the same prefix and Instructions, meanwhile their parent node is an empty one. 
Table 4: The Non-edge Flow Entries

\begin{tabular}{|c|c|c|}
\hline Priority & IP Address (last 8 bits in Binary) & Action \\
\hline 1 & $* * * * * 111$ & Green \\
\hline 2 & $* * * * * 110$ & Blue \\
\hline 3 & $* * * * * 001$ & Blue \\
\hline 4 & $* * * * * 000$ & Blue \\
\hline 5 & $* * * * * 11 *$ & Blue \\
\hline 6 & $* * * * * 01 *$ & Green \\
\hline 7 & $* * * * * 1 * *$ & Red \\
\hline
\end{tabular}

These three nodes could be replaced with a new one, which is exhibited in the Fig 4(a) as the intuitive reduction.

However, the efficiency of this intuitive reduction method is not satisfactory enough for its tight matching requirements. Especially in a vLAN (Virtual Local Area Network)[12] based SDN (mostly used in the data center), the terminals lose their regularity between the geographical positions and IP addresses.

Meanwhile, the intuitive reduction method experiences extended forwarding because it may ignore the possible empty nodes within the original sub-tree. When a flow with such IP address is discovered, it is automatically routed instead of be recognized as a new one. This is called the "extended routing".

In order to reduce the number of the non-empty nodes as much as possible and avoid the extended routing, we improve the intuitive reduction method with the idea shown as the Improved Reduction in Fig 4(a) \& (b). The figure is a sketch with an example of 3-digit addresses reduction. As indicated by the number attached to the edges, the address that a node represents is revealed by its location in the binary trie. For example, the node at the left bottom in the middle binary trie in Fig 4(a) represents an entry with an address as "000" and an action indicated by color "blue".

After the binary trie $T_{I B}$ generated, we supply the trie with additional nodes — ones with infinitely high priority and Instructions as "report to controller" - 
as siblings of non-empty single-child nodes. This operation is achieved by calling the Algorithm 4 Binary-Trie-Supplement $\left(T_{I B}\right)$. The graphical representation is presented as the Supplement module in Fig 4(b), and is also equivalently exhibited as the process between the former two lists of the flow entries in Fig 5.
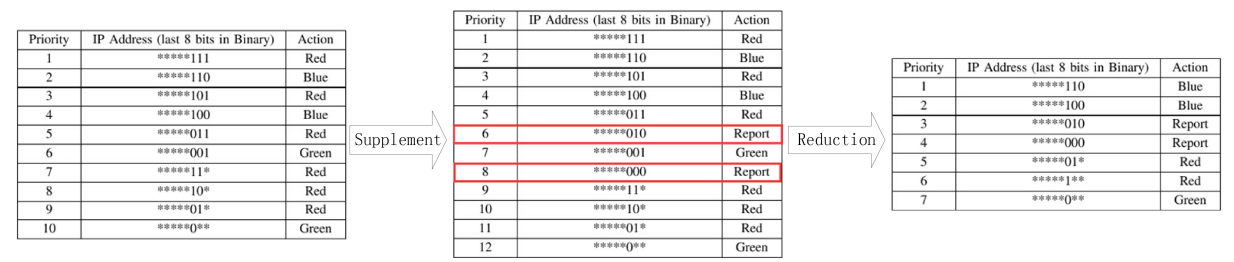

Figure 5: The Performance of FTRS in Fig 4(b)

Then, we check the all the nodes of the binary trie $T_{I B}$. If all the Instructions of the nodes within the sub-tree of a node are found the same (including itself), we take it as the representative of all the descendant nodes with the same action (or port). Otherwise we will find the mostly appeared action (or port) in the sub-tree and replace the root with the representative node. We delete all the nodes being represented next, and the address of nodes added by Algorithm 4 will be excluded from the Match Field of their non-empty ancestors by netmask at last. This process is exhibited as Algorithm 5 and as shown in Fig 5, the 1st, 3rd, 9th and 10th entries in the second list are replaced by the 4th entry in the third list. Similarly, the 5th and 7th entries are replaced by the original 11th and 12th entries in the second list. The equivalent process is also shown in Fig 4(b) as the Reduction module.

After the execution of the former 5 algorithms, the reduction is finished. Then, we restore the flow table from this binary trie, which is achieved by Algorithm 6. Similar with our former discussion on the priorities in the subtree, the entries should possess higher priorities than ones represented by their ancestors in the binary trie. In this case, the flow table is generated by the inverted sequence of the breadth first search (BFS) of the binary trie. The binary trie shown as the third graph in Fig 4(b) is transformed into a new flow 
table shown as the third list in Fig 5.

\subsection{Reduction on Other Attributes}

Besides the reduction on IP addresses, the other attributes shown in Fig 2(b) are also capable of reducing the number of flow entries. As we mentioned above, it is encouraged to make use of only one attribute for entry combination because there must be more entries to be matched with fewer attributes. Also, the multiple reduction is unnecessary, which is supported by our experimental results as well. There are situations that IP address involved attributes may not be suitable for reduction. For example, in a datacenter environment, which is a typical application scenario of SDN as well. The network is a vLAN, in which the IP addresses are mostly irregular among terminals and irrelevant with their physical locations. At this moment, the IP reduction may experience poorer performance than the rest attributes do. Typically, we test FTRS on "Ingress Port" in our experiment and the corresponding algorithms are shown as Algorithm $7 \&$ 8. Unlike the IP addresses involved attributes, the reduction on Ingress Port need not to consider the extended routing due to the fact that the ingress ports of a switch are mostly fixed and limited.

Algorithm 7 describes the intuitive thought to make flow entries combination on the typical number of Ingress Ports. It also suffers from the low efficiency just like Algorithm 3 does and we are able to design a more efficient one with the same idea of the improved IP reduction. The combination on port ID is too strict and we present a more flexible yet accurate reducing method in Algorithm 8. With the purpose of making maximal use of port matching, we transform ${ }_{85}$ the port IDs into binary strings and seek the similarities among them.

Then, the executing procedure of the port reduction is the same as the IP reduction, namely calling for Algorithm 2 for pruning the trie, Algorithm 4,5,6 for reducing the number of flow entries.

\subsection{Reduction Analysis}

590

There is only a "for" loop in algorithm 1 with the length of $n$ in line $2-5$, which contributes to its $O(n)$ complexity. It is the same for algorithm 6 (line 
$2-4$ ) and 8 (line $2-5$ ). In algorithm 7 there are two separate "for" loops - one with $n$ length (line $2-6$ ) and the other with a "no larger than $n$ " length (line 7 -11), which contribute to its $O(n)$ complexity. Algorithm 2 and 4 are both up-bottom self-calling algorithms, and only calculating with the values stored in the very node. Hence, their complexities depend on the size of the binary trie, namely the length of the flow table $n$, and are both $O(n)$.

On the other hand, algorithm 3 and 5 both check each node within the binary trie with a "for" loop (indicated as line $2-14$ and line $4-17$ respectively). However, within the loops, all the nodes within the subtree rooted by the node are also checked (line $4-13$ and line $5-16$ respectively), which contributes to their $O(n \operatorname{lgn})$ complexity. Hence, the total calculating complexity of our reduction is $O(n \lg n)$.

Due to the fact that the IP reduction is the most efficient and the IP addresses are completely different in LAN and vLAN, we have to decide the algorithm executing timing and conditions. As we stated before, in a vLAN the IP addresses are irregular, which makes the IP reduction nearly ineffective. While on the contrary, the rest attributes such as Ingress Port are not influenced. They experience mediocre but better-than-IP-address performance in the vLAN environment.

The feasibility of the newly generated flow table is ensured since we only combine the original entries into coarse-grained new ones. The original flow routing function is absolutely not influenced, and our improved reduction method guarantees the flow discovery function. The preservation of the Terminal entries also ensure the data collecting functions of SDN. The controller's functions are guaranteed in FTRS.

\subsection{The Process after Reduction}

Since the network conditions are mostly changed with the time, we should ensure the network is functioning normally and the combined entries won't impact the the network functions if they are not suitable to the network conditions after the reduction. The normal functionality of SDN is based on the flow entry. 
So we have to make sure the flow entry involved operations are not influenced by FTRS.

As we stated above, the original functions of SDN are ensured by our design, functionality of SDN.

\section{SIMULATION \& EXPERIMENT}

In this section, we present the performance of FTRS both in simulation and experiment. We firstly present a brief description on our simulation method and exhibit the experimental results on different parameters. Besides simulation, a small-scale test-bed experiment is introduced and we examine the performance of multiple reduction with contrast experiments. Our conclusion on optimizing objectives is given at last. 


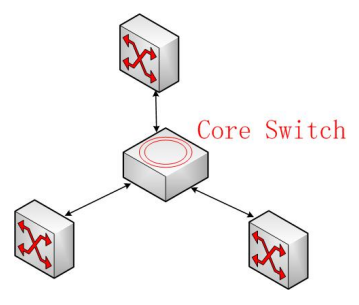

(a) Star Topology

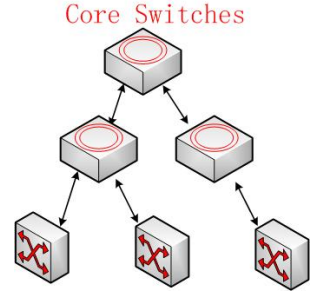

(b) Tree Topology

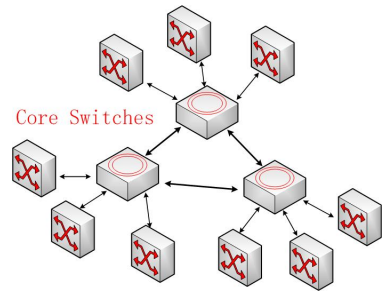

(c) Distributed Topology

Figure 6: Three kinds of Topology

\subsection{Simulation Method}

We evaluate the performance of FTRS with the help of Mininet[14], which is a typically designed tool for SDN simulation. Mininet simulates the elements and operation functions of SDN with reliable performance and is widely used in SDN research.

In our simulation, the star, tree and distributed topologies which are three representations in LAN deployment are examined with our algorithms running on the switches in it. The circle topology is also popular but barely used in SDN deployment. As a result, it is not considered in our experiment. The network topologies are shown in Fig 6 and we monitor the number of flow entries in the flow tables of all the switches in the simulation.

The topology scale in our simulation is mostly no more than 200 elements in one network both due to the capacity limitation of Mininet and practical experience. Particularly, the range size of star, tree and distributed topologies are 31-105, 23-149 and 30-201 respectively. We simulate the performance of each network topology for ten times under different scales.

Because we are the first to focus on reducing the number of flow entries and manage to preserve SDN functionality, which have not been achieved by any current technique yet, we analyze our performance by comparing with the original network conditions instead of any other techniques. 


\subsection{Evaluation Parameters}

In order to evaluate the performance of FTRS, we arrange several parameters to analyze and represent the performance.

Firstly, the efficiency of reduction is represented by the percentage $p$, which is defined as the number of flow entries that are reduced to the number of original flow entries.

Meanwhile, we calculate the ratio between the number of flow entries in the Core Switch and that in the Boundary Switch, which is formulated as $c_{r}$ the Congestion Ratio. This parameter helps in both exhibiting the reduction efficiency and revealing the network resource allocation.

680

Similar to the example shown in Fig. 3, we are able to calculate the optimal situations under different topologies regardless of the fine-grained characteristic. The calculation is necessary to a better understanding of the performance evaluation in the following.

It is obvious that the star topology is a particular case of distributed topology, so we formulate the performance in the tree and distributed topologies separately in this part.

In a distributed topology, we assume that there are $k$ core switches, $n * k$ normal switches and $m * n * k$ terminals with equal distribution for simplification. When all the computers in network are able to communicate with each other, the number of flow entries in the flow tables of Boundary Switch $\left(\mathcal{S}_{\text {boundary }}\right)$ and Core Switch $\left(\mathcal{S}_{\text {core }}\right)$ can be calculated by Formula (1).

$$
\begin{gathered}
\mathcal{S}_{\text {boundary }}=m(2 k m n-m-1) \\
\mathcal{S}_{\text {core }}=m^{2} n(2 k n-n-1)
\end{gathered}
$$

According to Formula (1), the least number of flow entries existed in Boundary and Core Switch are 2148 and 8064 in a distributed network with 1 Core Switch, 8 Boundary Switches and 96 terminals - namely $k=1, n=8, m=12$.

Meantime, the number of flow entries in the Core Switch is able to be reduced to $n+k-1$ optimally in FTRS. 
In this case, in a distributed network, the congestion ratio $\left(c_{r}\right)$ and the optimal reduction percentage $\left(p_{\text {opt }}\right)$ are calculated in Formula (2).(In the case we proposed, these two parameters are 6.04 and $99.864 \%$ respectively)

$$
\begin{gathered}
c_{r}=\frac{m n(2 k n-n-1)}{2 k m n-m-1} \\
p_{\text {opt }}=1-\frac{n+k-1}{m^{2} n(2 k n-n-1)}
\end{gathered}
$$
addresses reduction in the flow table in a simulated LAN.

In the simulation of IP addresses FTRS, we arrange 10 different scales for each topology separately in the simulation, and experimental results are shown 


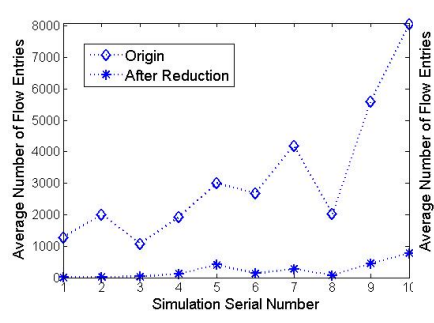

(a) Star Topology

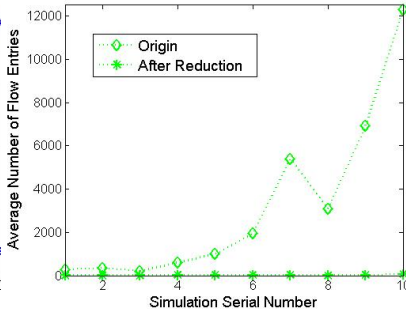

(b) Tree Topology

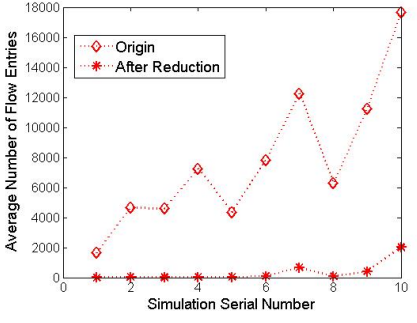

(c) Distributed Topology

Figure 7: The Reduction Performance at the Core Switch

in Fig 7(a)(b)(c). In each type of topology, the parameter $n$ and $m$ are always 5

In order to analyze the influence to the controller's function through FTRS, we analyze the existence of all the flow entries by checking whether their monitoring data are available after reduction. This is also a simulation to the support 

sibility that some of the entries may be deleted automatically for not matched after a period of time, these entries may become not available. However, in practice, it is impossible that all the flow entries are added by applications they usually take only a tiny part of the existed entries. In this case, our simThe results are shown in Fig 8(b). FTRS both achieves perfect performance in star and tree topologies. Meanwhile, more than $90 \%$ of the entries are available on average in the distributed topology. Notably, the not available entries are deleted automatically during the operation of FTRS, indicating they are not matched for a period of time. Their corresponding flows will be routed instead of recognized as a new flow. However, this is hardly to happen, because these flows will be reported to the controller by the Boundary Switch before they reach the Core Switch. In this case, the normal network transmission is actually not influenced at all.

It could be tiny but should not be huge. This is because the flow entries do not congested in Boundary Switch but in Core Switch. As shown in Fig 8(c), $c_{r}$ drops below 0.3 after the reduction, indicating the number of flow entries in Core Switch is much smaller than that in Boundary ones. It leaves great potential for the network to service more new flows with QoS guaranteed.

\subsection{Reduction Simulation on Other Attributes}

Since the IP addresses reduction may experience poor performance in some typical situations, such as a vLAN, in which the IP addresses are irregular and irrelevant with the topology, to seek the possibility of reduction on other attributes is necessary.

\subsubsection{The vLAN Environment}

Unlike a LAN, vLAN is mostly used in the network made up with virtual machines (VMs). Although these VMs generally share neighboring physical 


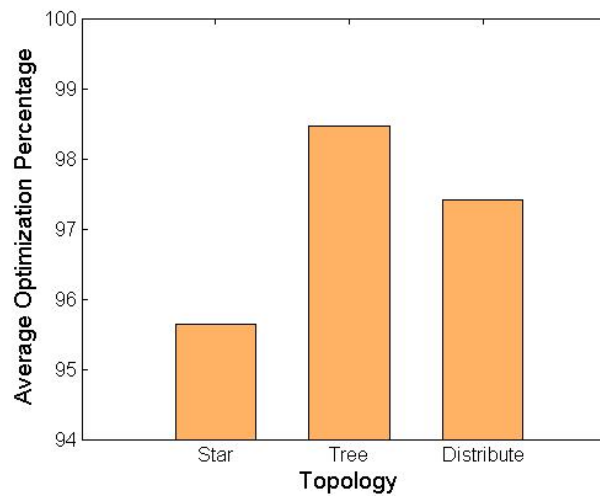

(a) Performance of IP Reduction

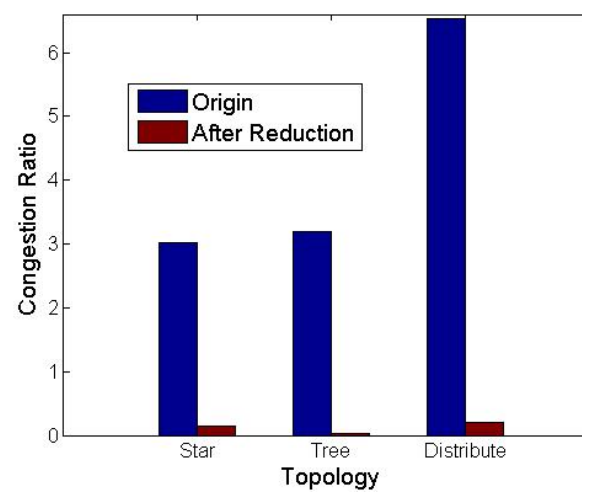

(c) Congestion Ratios

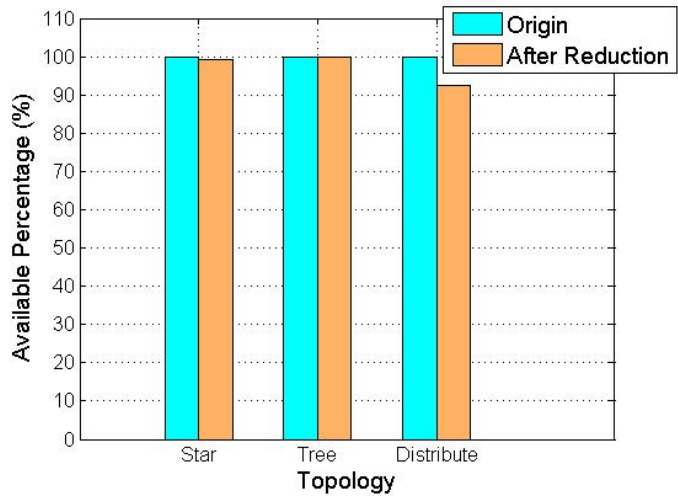

(b) Percentage of Available Entries

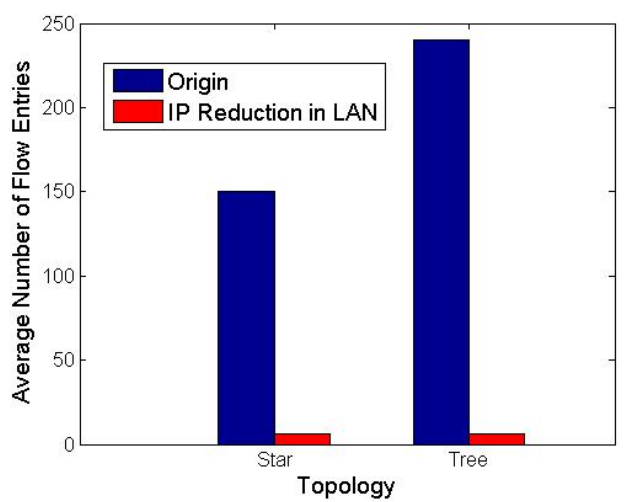

(d) Practical Data

Figure 8: IP Reduction Simulation \& Experimental Data

carriers, their IP addresses are in chaos. SDN products are also widely used in vLAN in industry. They mostly use the Open vSwitch (OVS)[21] to route the packets among VMs. Although possesses the unlimited flow table storage in theory, OVS still requires reducing the number of flow entries. This is because as a software OpenFLow switch, OVS performs poor in one of the core promise of SDN - the fast forwarding, and the situation is made even worse if it takes too much time to go through the flow table due to the large amount of entries. However, the IP reduction may experience badly performance in a vLAN. With the purpose of reducing the number of flow entries in OVS, the possibility to 


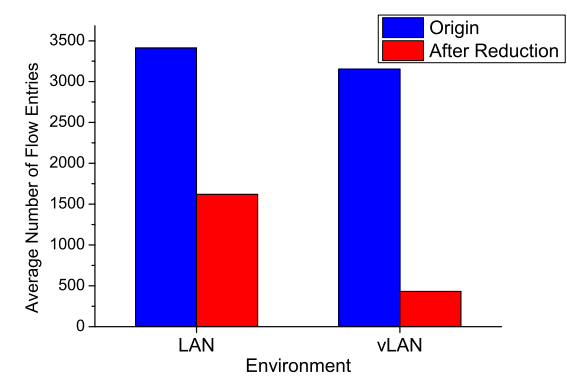

(a) Port Reduction

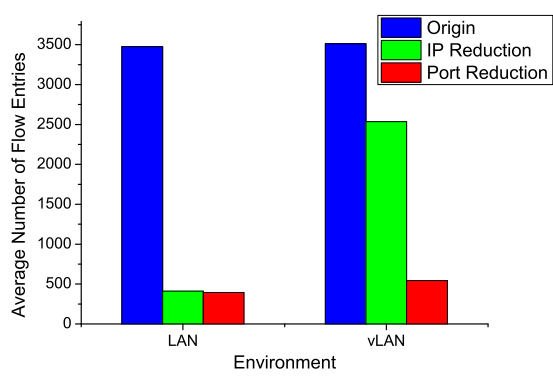

(b) Multiple Reduction

Figure 9: The Port/Multiple Reduction Performance

use other attributes in the Match Field in FTRS should be considered and examined.

\subsubsection{Reduction on other Objective}

All the attributes shown in Fig. 2 are able to be used to reduce the number of flow entries. Since the Ingress Port is not in chaos as the IP address, this attribute helps to indicate the transmission direction logically. In this case, we make a comparative trial on the reduction performance on Ingress Port both in a $k=1, n=3, m=6$ LAN of star topology and a vLAN with 1 OVS, 18 VMs. They share the same number of terminals and in turn similar original number of flow entries. The results are exhibited in Fig 9(a). The reduction on Ingress Port experiences unspectacular performance in a LAN environment and much better one in a vLAN. This is mainly due to the port is limited physically in LAN but defined by the software in vLAN. OVS not only possess unlimited number of port but also define them in its own way. As a result, it is much easier to seek similarity among the Ingress Ports in OVS than in physical OpenFlow switch. Although the performance is not as spectacular as the IP reduction, to reduce the number of flow entries in OVS prefers Ingress Port instead of IP address. This is revealed in the later multiple reduction results shown in Fig. 9(b). 


\subsubsection{Feasibility 83 Necessity discussion of Multiple Reduction}

As we mentioned before, the reductions on various objectives are capable of superposition but not necessary. In this part, we present the simulation

\subsection{Test-Bed Experiment}

A practical experiment is implemented to verify our estimation and simulation of our former discussion. Our detailed implementation is listed below.

\subsubsection{Controller Settings}

A server with a Linux OS (ubuntu 12.04) undertakes the responsibility of 825

multiple reduction. In most of the cases, IP reduction is efficient enough to solve FTCP. When the flow table is not congested, we need not and should not keep on reducing the number of the entries since the fine-grained entries are at least helpful in management and data collection. FTRS trades off part of the not important fine-grained entries storage to achieve the robustness of SDN services and it is not necessary to seek the perfect reduction efficiency. Moreover, although experiencing acceptable time complexity, FTRS takes up time for calculating, analyzing and flow table transmitting. If the network is not steady, it is meaningless to call for several reductions due to the fact that if the network is steady, is it necessary to call for multiple reduction? With the question we test the performance of FTRS for twice reduction. We use one OpenFlow switch/OVS with several terminals linked to it. Each of the terminals generates several steady flows during the simulation. As a result, the flow tables are steady. FTRS uses IP address and Ingress Port for reduction in sequence. The simulation results are shown in Fig 9(b). It is obvious that the Port reduction performs almost uselessly after the IP reduction in a LAN environment and the IP reduction performs ineffectively in a vLAN environment. As a conclusion, the multiple reduction is unnecessary in FTRS.

\footnotetext{
controller in our experiment. We used a specifically designed OpenFlow manage-
} 
ment open-source program "OpenDayLight" [19] as the controller in the server. The typical vision of our OpenDayLight is 1.3 and some modifications are made to ensure the normal operation.

\subsubsection{OpenFlow Switches}

Four commercial OpenFlow switches [20] are used as core and normal switches in our deployed SDN. The manufacturers are concealed by fuzzy processing. These switches submit the OpenFlow protocol 1.0.

\subsubsection{Network Terminals}

We arranged 20 personal computers connected with OpenFlow switches with

835 net as usual and share files within the LAN at the same time.

As we discussed in Part $\boldsymbol{B}$, the optimal Congestion Ratio $c_{r}$ and Reduction Percentage $p_{\text {opt }}$ are 1.24 and $98.61 \%$ respectively. In our test-bed experiment, the actual data are 1.31 and $95.07 \%\left(\frac{186}{142}\right.$ and $\left.\frac{135}{142}\right)$. The Congestion Ratio is the flow entries storage. Meanwhile, the Reduction Percentage is slightly lower than the optimal value but still remarkable. The performance of FTRS hardly achieves the optimal result because the flow entries are various and not all of them could be combined. spectively. Due to the limited number of switches, we omitted the experiment of distributed topology but took the star topology as its special case. The terminals are located almost equally in both environments. The communication operation between terminals is achieved with the help of "ping" command. The performance of our test-bed is shown in Fig 8(d).

It is shown from the figure that FTRS experiences an outstanding performance in a LAN environment just as we estimated. Due to the lack of experimental devices, we are only able to examine the performance of FTRS with a small quantity of flow entries. Since we use "ping" command as the communica- 


\section{Conclusion}

In this paper, we have presented a novel mechanism Flow Table Reduction Scheme (FTRS) to deal with the Flow Table Congestion Problem (FTCP) in SDN. We have proposed detailed discussion on the origin of the problem, its influence on network functions and the importance and urgency to solve it. Several algorithms have been proposed in our carefully designed scheme to reduce the number of flow entries with SDN normal functionality guaranteed. FTRS is able to reduce the number of flow entries by more than $95 \%$ generally in LAN and also remarkable performance in vLAN. The possibility and feasibility of using FTRS in other environments are also confirmed in our discussion. FTRS is proved to be suitable for long time operating in SDN as well.

The simulation and experiments have been made with minces data and the results confirm our inference.

We will try to find an approximation algorithm for the "Flow Table Congestion Problem" as a future work. We will also seek for other ways for flow entry reduction that is even harmless to the unnecessary entries and guarantees the full functionality of SDN in the future.

\section{Acknowledgment}

This paper is supported by the National Science Foundation of China under No. U1301256, 61272133, and 61472383, Special Project on IoT of China NDRC

${ }_{880}$ (2012-2766), Research Fund for the Doctoral Program of Higher Education of China No. 20123402110019. 


\section{References}

[1] B. Leng, L. Huang, X. Wang, H. Xu, Y. Zhang, A mechanism for reducing flow tables in software defined network, in: 2015 IEEE International Conference on Communications (ICC), IEEE, 2015, pp. 5302-5307.

[2] N. McKeown, T. Anderson, H. Balakrishnan, G. Parulkar, L. Peterson, J. Rexford, S. Shenker, J. Turner, Openflow: enabling innovation in campus networks, ACM SIGCOMM Computer Communication Review 38 (2) (2008) 69-74.

[3] B. K. Mathew, N. A. Issac, Ternary content addressable memory.

[4] A. R. Curtis, J. C. Mogul, J. Tourrilhes, P. Yalagandula, P. Sharma, S. Banerjee, Devoflow: scaling flow management for high-performance networks, ACM SIGCOMM Computer Communication Review 41 (4) (2011) 254-265.

[5] M. Yu, J. Rexford, M. J. Freedman, J. Wang, Scalable flow-based networking with difane, ACM SIGCOMM Computer Communication Review 40 (4) (2010) 351-362.

[6] Y. Kanizo, D. Hay, I. Keslassy, Palette: Distributing tables in softwaredefined networks, in: INFOCOM, 2013 Proceedings IEEE, IEEE, 2013, pp. 545-549.

[7] B. A. A. Nunes, M. Mendonca, X.-N. Nguyen, K. Obraczka, T. Turletti, A survey of software-defined networking: Past, present, and future of programmable networks, IEEE Communications Surveys \& Tutorials 16 (3) (2014) 1617-1634.

905

[8] L. Liu, R. Muñoz, R. Casellas, T. Tsuritani, R. Martínez, I. Morita, Openslice: an openflow-based control plane for spectrum sliced elastic optical path networks, Optics express 21 (4) (2013) 4194-4204. 
[9] M. G. Gouda, X.-Y. Liu, Firewall design: Consistency, completeness, and compactness, in: Distributed Computing Systems, 2004. Proceedings. 24th International Conference on, IEEE, 2004, pp. 320-327.

[10] M. Yoon, S. Chen, Z. Zhang, Minimizing the maximum firewall rule set in a network with multiple firewalls, IEEE Transactions on Computers 59 (2) (2010) 218-230.

[11] N. Kang, Z. Liu, J. Rexford, D. Walker, Optimizing the one big switch abstraction in software-defined networks, in: Proceedings of the ninth ACM conference on Emerging networking experiments and technologies, ACM, 2013, pp. 13-24.

[12] Openflow, Openflow - enabling innovation in your network, http://www.openflow.org/ (retrieved 2016).

[13] S. Das, Y. Yiakoumis, G. Parulkar, N. McKeown, P. Singh, D. Getachew, P. D. Desai, Application-aware aggregation and traffic engineering in a converged packet-circuit network, in: National Fiber Optic Engineers Conference, Optical Society of America, 2011, p. NThD3.

[14] A. Mimidis, C. Caba, J. Soler, Dynamic aggregation of traffic flows in sdn: Applied to backhaul networks, in: NetSoft Conference and Workshops (NetSoft), 2016 IEEE, IEEE, 2016, pp. 136-140.

[15] X.-N. Nguyen, D. Saucez, C. Barakat, T. Turletti, Rules placement problem in openflow networks: a survey, IEEE Communications Surveys \& Tutorials 18 (2) (2016) 1273-1286.

[16] B. Stephens, A. Cox, W. Felter, C. Dixon, J. Carter, Past: Scalable ethernet for data centers, in: Proceedings of the 8th international conference on Emerging networking experiments and technologies, ACM, 2012, pp. 4960.

[17] R. Wang, D. Butnariu, J. Rexford, et al., Openflow-based server load bal935 ancing gone wild., Hot-ICE 11 (2011) 12-12. 
[18] H3c communication \& technology limited company, http://www.h3c.com.cn/.

[19] J. Medved, R. Varga, A. Tkacik, K. Gray, Opendaylight: Towards a modeldriven sdn controller architecture, in: A World of Wireless, Mobile and Multimedia Networks (WoWMoM), 2014 IEEE 15th International Symposium on, IEEE, 2014, pp. 1-6.

[20] H3C, H3c s6300-f configuration wizard, http://www.h3c.com.cn/Service/Document_Center/Switches/Catalog/S6300/S6300F/Configure/Operation_Manual/H3C_S6300-F_CG-Release_23106W100/14/201406/833079_30005_0.html (retrieved 2016).

[21] B. Pfaff, J. Pettit, K. Amidon, M. Casado, T. Koponen, S. Shenker, Extending networking into the virtualization layer., in: Hotnets, 2009.

[22] K. K. Chan, P. W. Hartmann, S. P. Lamons, T. G. Lyons, A. C. Milonas, Virtual local area network, uS Patent 4,823,338 (Apr. 18 1989).

[23] H. Samet, The design and analysis of spatial data structures, Vol. 199, Addison-Wesley Reading, MA, 1990.

\section{Appendix}

Algorithms 


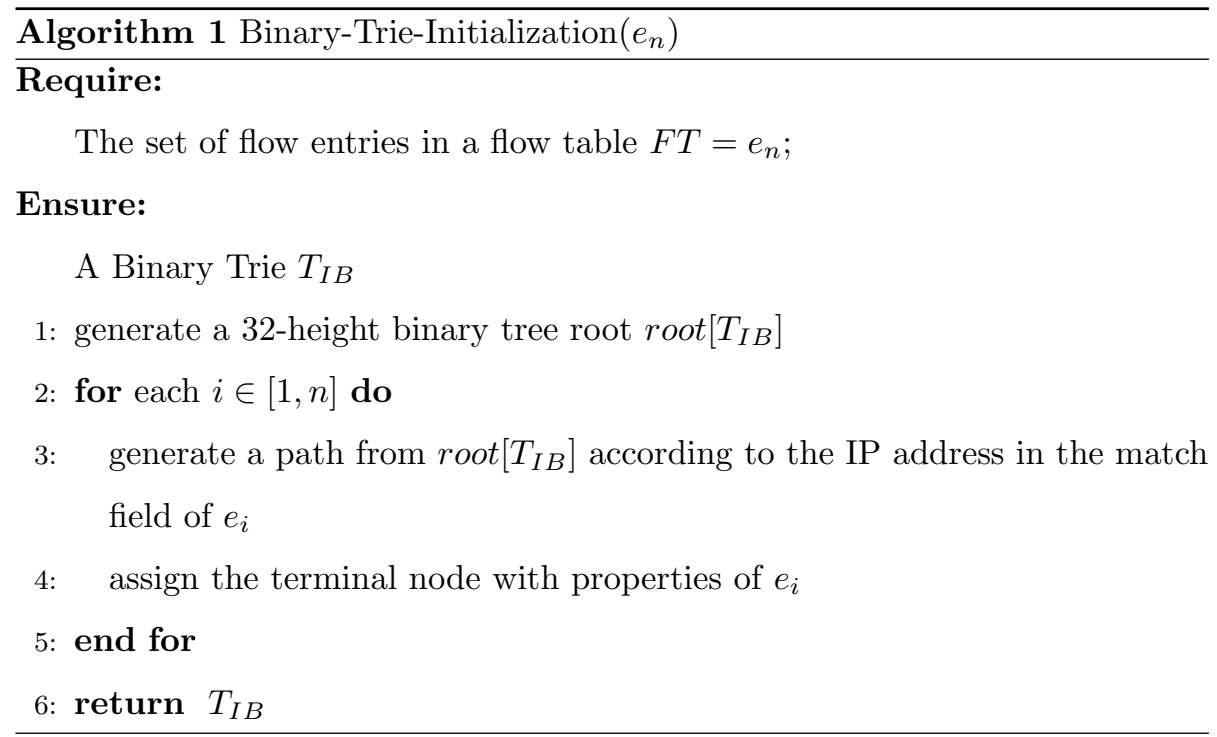




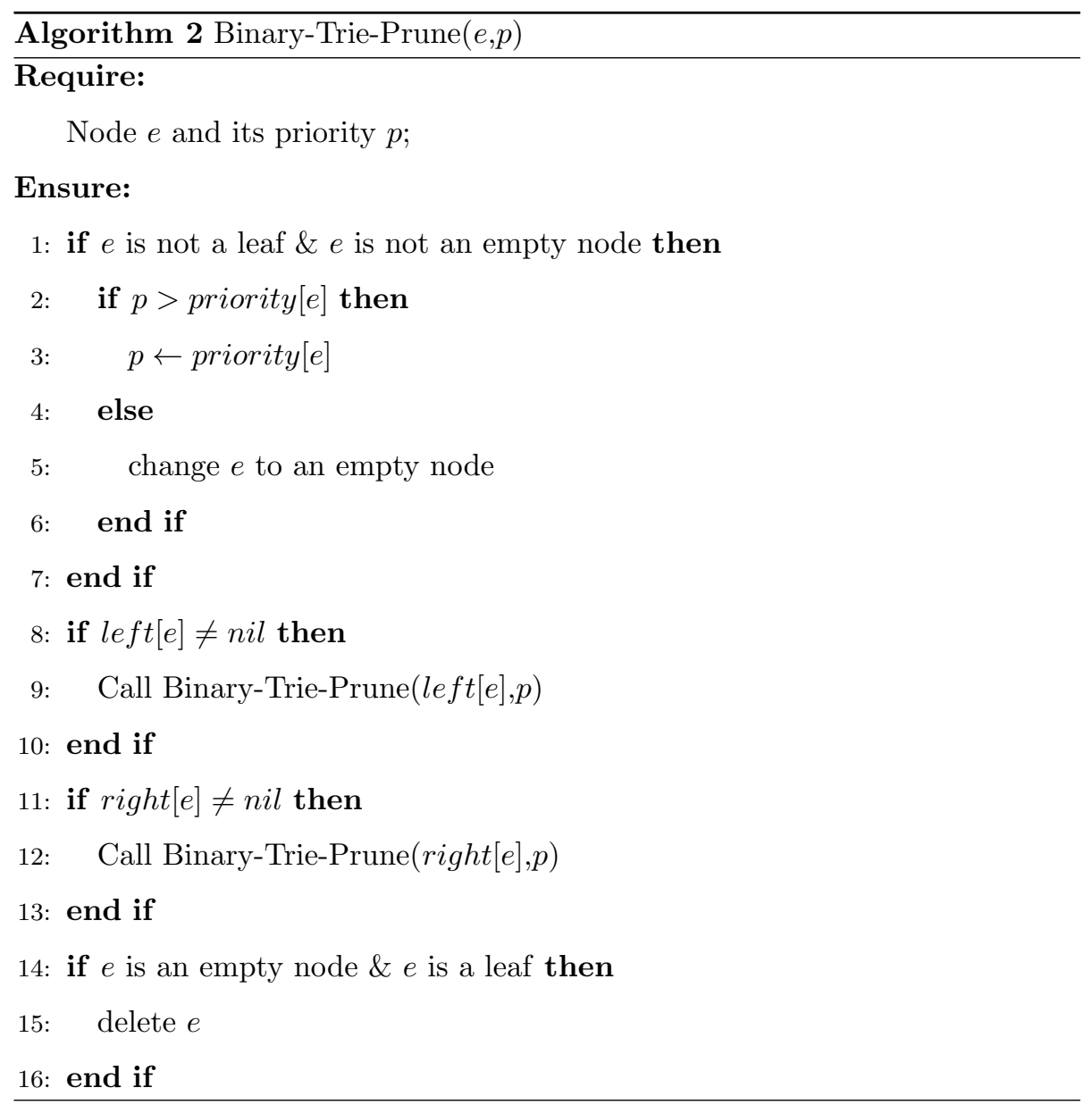




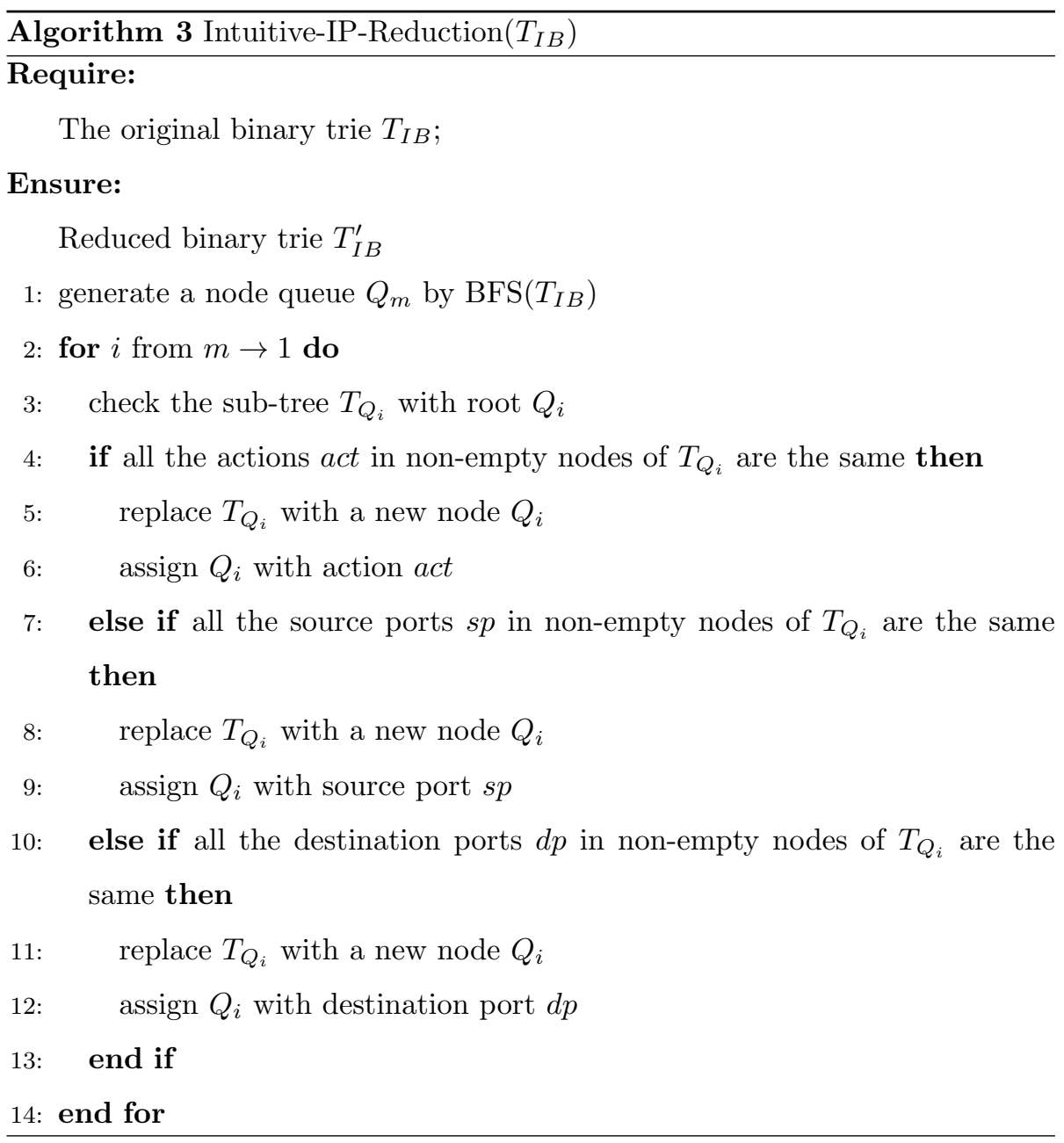




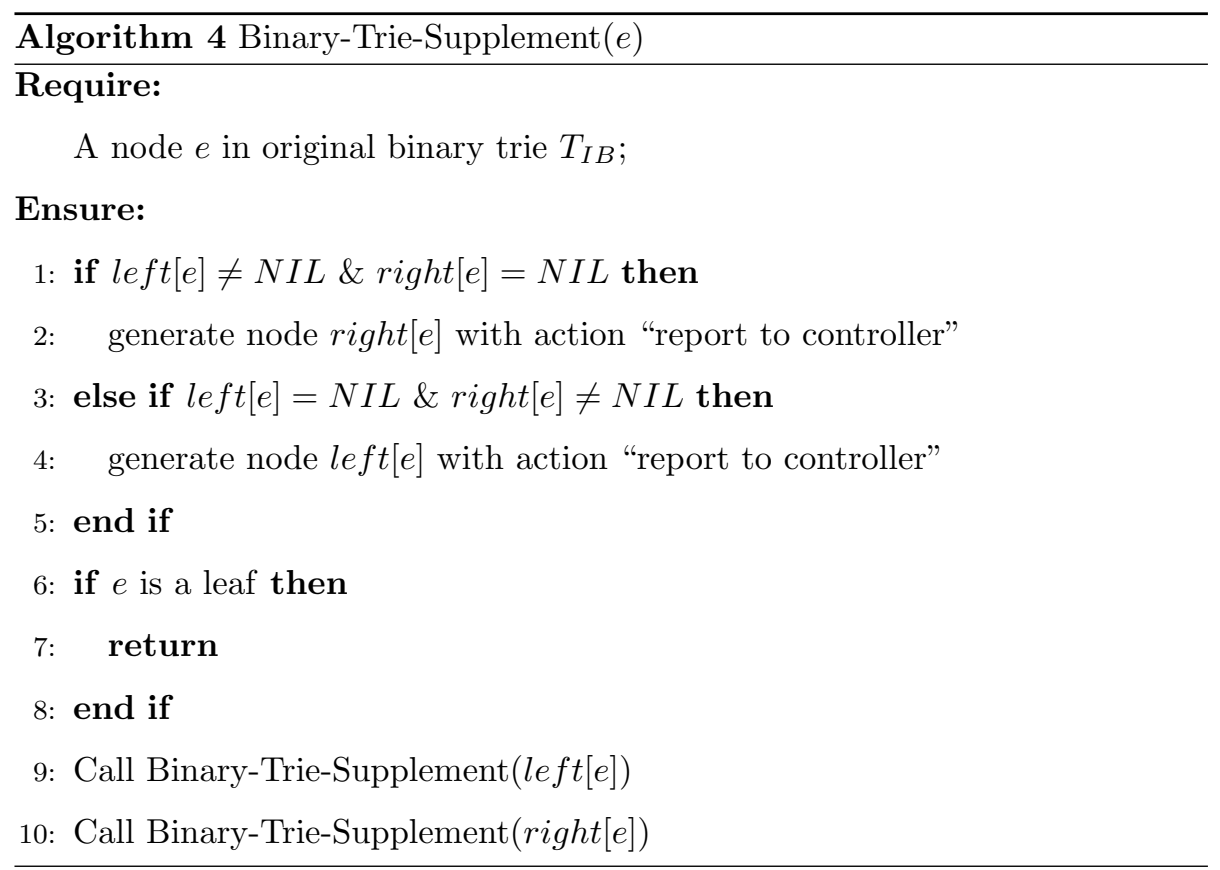




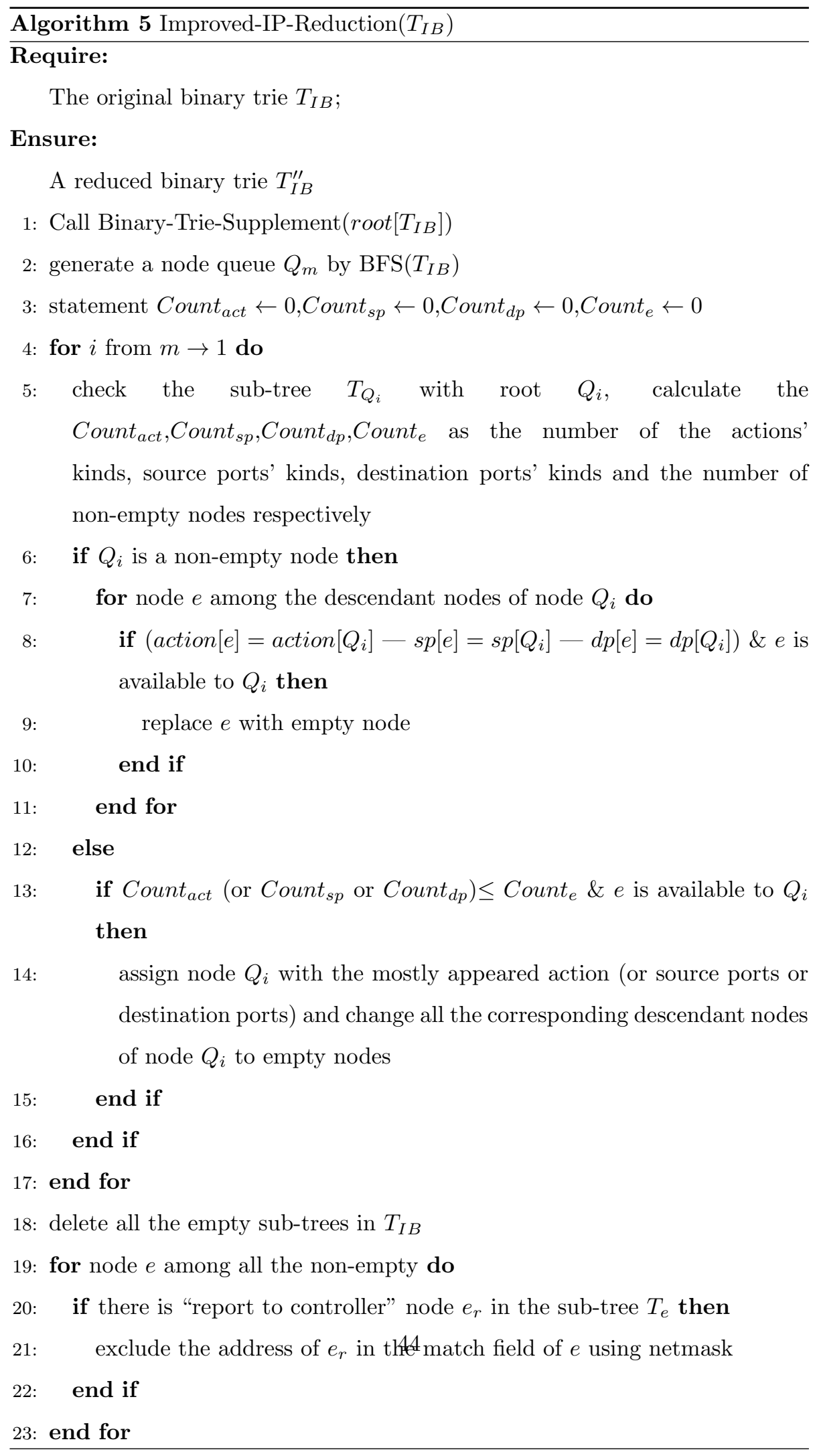




\begin{tabular}{l}
\hline Algorithm 6 Flow-Table-Restore $\left(T_{I B}\right)$ \\
Require: \\
$\quad$ A binary trie $T_{I B} ;$ \\
Ensure: \\
$\quad$ A flow table $e_{T_{I B} . \text { size }}$ \\
1: generate a non-empty node queue $Q_{m}$ by $\operatorname{BFS}\left(T_{I B}\right)$ \\
2: for $i$ from $m \rightarrow 1$ do \\
3: $\quad$ write the flow entry $e_{i}$ with properties of $Q_{i}$ \\
4: end for
\end{tabular}

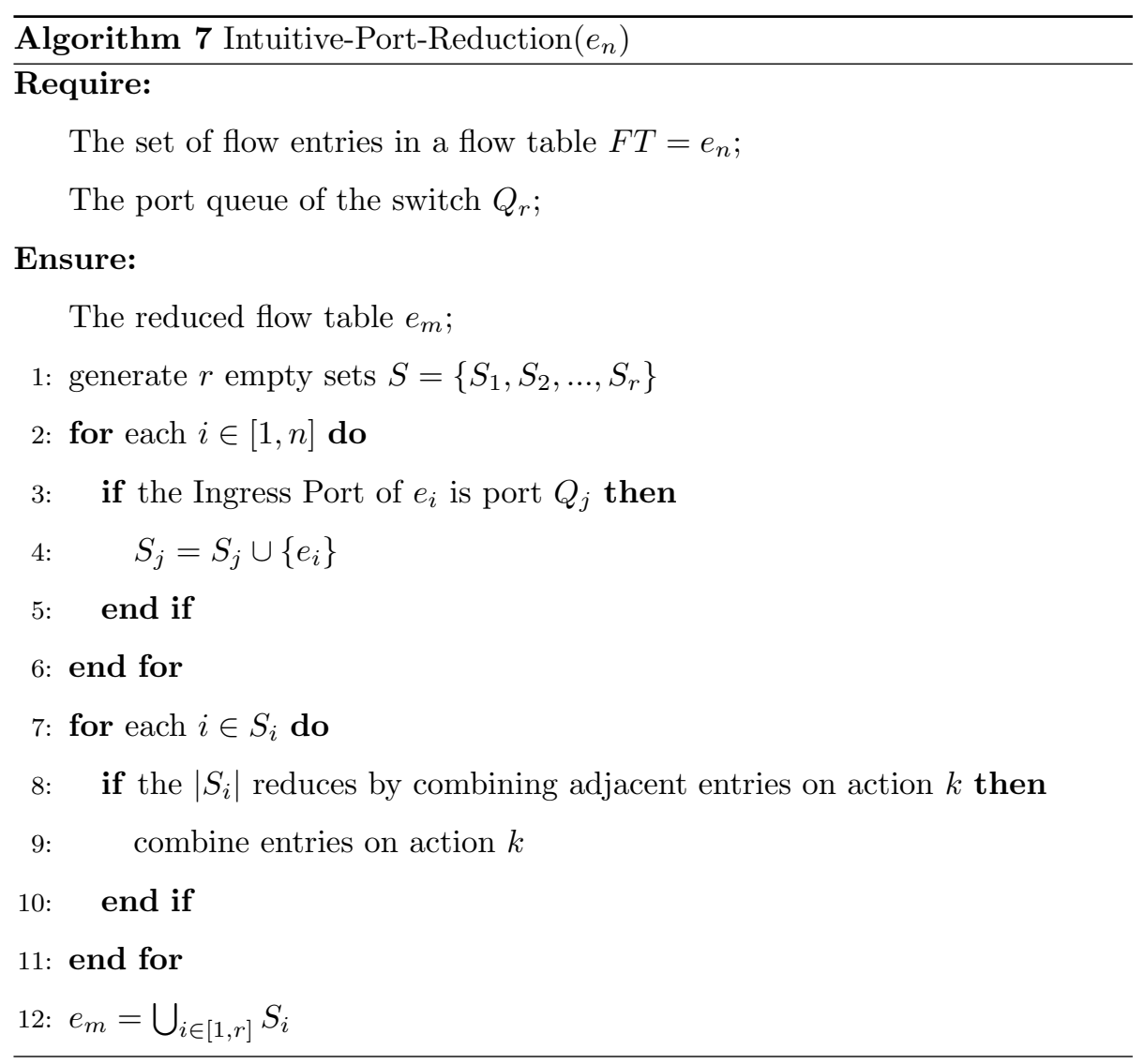




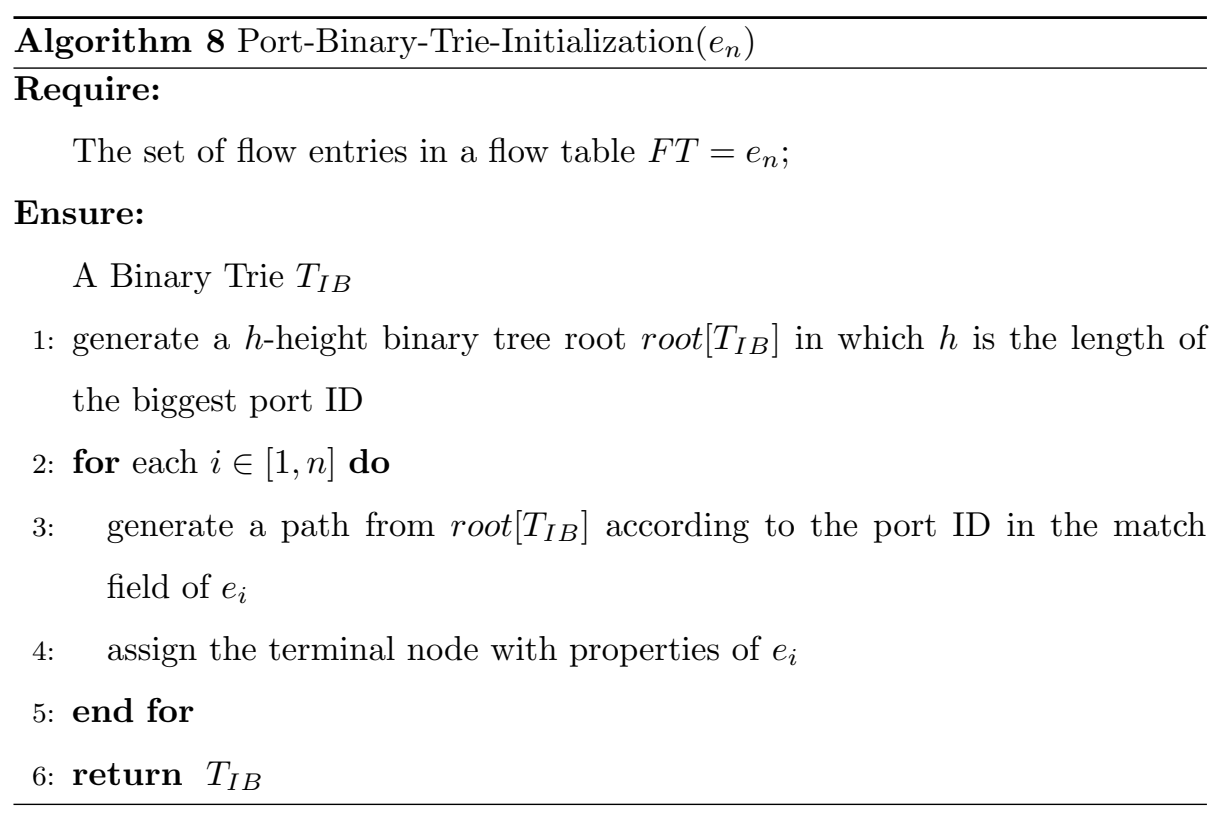


1

2
Bing Leng received the bachelor degree in 2012 from University of Science and Technology of China (USTC). He is currently a Ph.D student in the School of Computer Science and Technology, University of Science and Technology of China (USTC), Anhui, China. His research expertise and interests include Software-defined Network, Wireless Mesh Network, Smartphone-based Sensing and Applications.

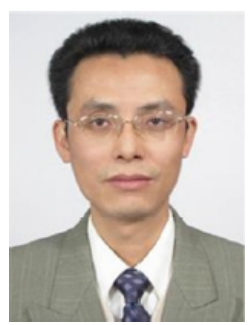

Liusheng Huang was born in Anhui Province, China, in 1957. He received the M.S. degree in computer science from University of Science and Technology of China in 1988. He is currently a professor and $\mathrm{Ph} . \mathrm{D}$. supervisor of the School of Computer Science and Technology at the University of Science and Technology of China. He has published six books and more than 200 papers. His research interests are in the areas of internet of thing, wireless sensor networks, information security and big data.

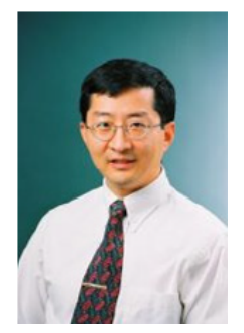

Chunming Qiao directs the Lab for Advanced Network Design, Analysis, and Research (LANDER) at SUNY Buffalo. He is a recipient of many awards including the SUNY Chancellors Award for Excellence in Scholarship and Creativity. He has published many highly cited papers with an h-index of over 60 (according to Google Scholar). He has seven US patents. He pioneered research on Optical Internet in 1997, and one of his papers has been cited more than 2,000 times. He also pioneered the work on integrated cellular and ad hoc relaying systems (iCAR) in 1999, which is recognized as the harbinger for todays push towards the convergence between heterogeneous wireless technologies, and has been featured in BusinessWeek and Wireless Europe, as well as at the websites of New Scientists and CBC. These works have inspired a lot of follow-up works around the world and attracted funding from a dozen of major IT and telecommunications companies including Alcatel, Cisco, Google, NEC labs, Nokia, and Telcordia.

Dr. Qiao has given a dozen of keynotes, and numerous invited talks on the above research topics. He has chaired and co-chaired a dozen of international conferences and workshops. He was an editor of several leading IEEE journals, and chaired a number of technical committees. He was elected to IEEE Fellow for his contributions to optical and wireless network architectures and protocols.

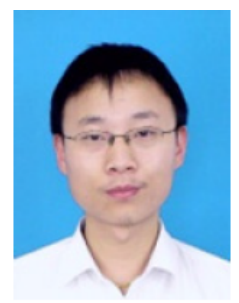

Hongli Xu received his $\mathrm{Ph}$. D degree in Computer Science from the University of Science and Technology of China in 2007. Currently, he is an associate research fellow in the School of Computer Science and Technology at the University of Science and Technology of China. His main research interest is cooperative communication, vehicular ad hoc network and software defined networks.

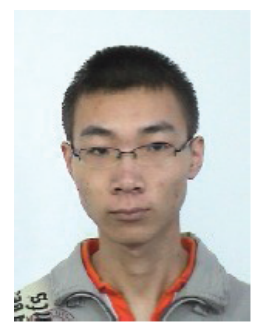

Xinglong Wang is currently a doctor-candidate in Computer Science at the University of Science and Technology of China. He will receive the doctor degree in 2017. His main research interest is wireless MIMO ad hoc networks. 
${ }^{*}$ Author Photo
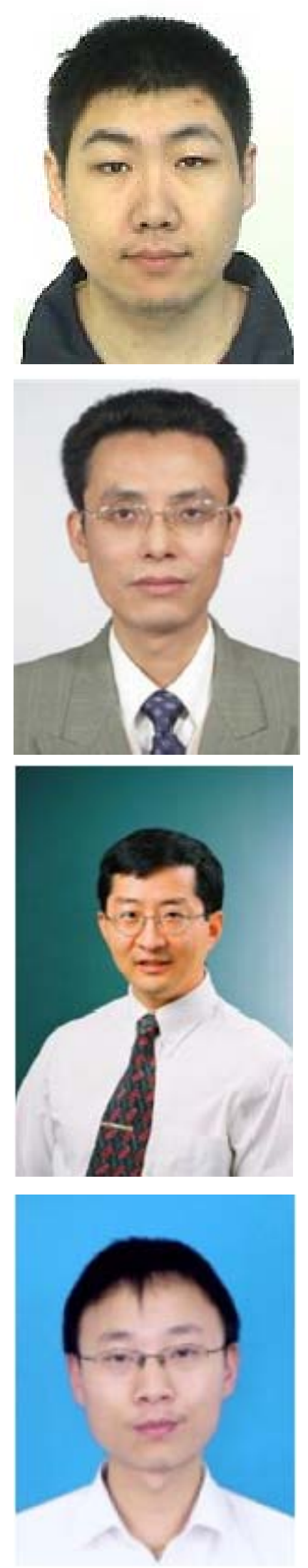


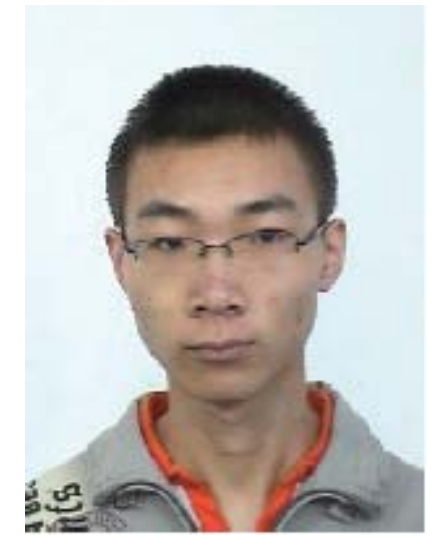

